\title{
Operative Precept of robotic arm expending Haptic Virtual System
}

\author{
Arnab Das ${ }^{1}$, Swagat Kumar Samantaray ${ }^{2}$, Anil Kumar Patra ${ }^{3}$ \\ ${ }^{1,2}$ (M.Tech-CSE, $2^{\text {nd }}$ year), Assistant Professor, National Institute of Science and Technology \\ ${ }^{3}$ (B.Tech-EIE, 3rd year)
}

\begin{abstract}
This paper presents the overview of a robotic arm with fingers having all features like a normal human and can do all operation that the human hand can do. It has 5 fingers and normal joints as that of a human. Robotic arm is made of by using Haptic Technology. 'Haptic' is a technology that adds the sense of touch to virtual environments or mechanical models. A haptic device gives people a sense of touch with computer-generated or real environments, so that when objects are touched, they seem real and tangible. In this paper we are describing about robotic arm which is controlled by the human hand movement so that the arm can reach the target location and perform the desired task. The arm will be controlled by Potentiometers, Accelerometer Sensor and Flex Sensor. This paper describes about how sensors are used for tracking the position and movement of the robot arm. The user wears a cyber gloves which are fitted with the above tactile sensors. Usually this sensor gives analog output, if there is a change in their resistance value. This analog output is manipulated in such a way that they give proportional pulses of different duty cycles to the servo motors which give movement to the robotic arm.
\end{abstract}

Keywords-Haptic system, Servo Motor, Flex Sensors, Accelerometer Sensor,kinematics.

\section{INTRODUCTION}

Haptic technology refers to technology that interfaces the user with a virtual or real environment via the sense of touch by applying forces, vibrations, and/or motions to the user. This mechanical stimulation may be used to assist in the creation of virtual objects existing only in a computer simulation, for control of such virtual objects, and to enhance the remote control of machines and devices [1][2]. Haptic interfaces presenting force and tactile feeling at human fingertips are used in the area of tele-manipulation of robots, simulation and design in virtual reality environments, educational training, and so on. Multi-fingered haptic interface is required to be safe, to work in wide operation space, and to present not only force at contact points but also weight feeling of virtual objects[3], to have no oppressive feeling when it is attached to humans, and to have no weight feeling of itself [4]-[6]. The control methods are well suited not only for the multi-fingered haptic interface but also for the multi-fingered robot hand for grasping and manipulating an object [6]. This emerging technology promises to have wide-reaching applications as it already has in some fields [7]. For example, haptic technology has made it possible to investigate in detail how the human sense of touch works by allowing the creation of carefully controlled haptic virtual objects. These objects are used to systematically probe human haptic capabilities, which would otherwise be difficult to achieve. These new research tools contribute to our understanding of how touch and its underlying brain functions work. Haptic devices are capable of measuring bulk or reactive forces that are applied by the user; it should not to be confused with touch or tactile sensors that measure the pressure or force exerted by the user to the interface.

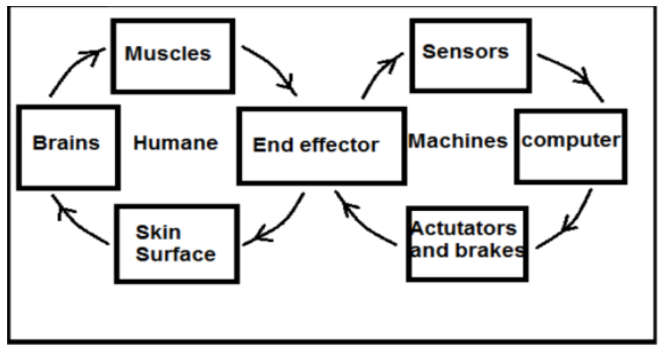

Fig. 1 haptic system

Haptic may be said as its mutual relation between the human and machine. Here parts of human senses and controls the position of hand, while the machine part exerts forces from the hand to stimulate contact with a 
virtual object. The both systems are provided with sensors, processors and actuators. Here in human system nerve receptors performs sensing, brain performs processing and muscles perfumes actuation of the motion performed by hand, while in case machine the function are performed by encoder, computer and motors respectively[8].

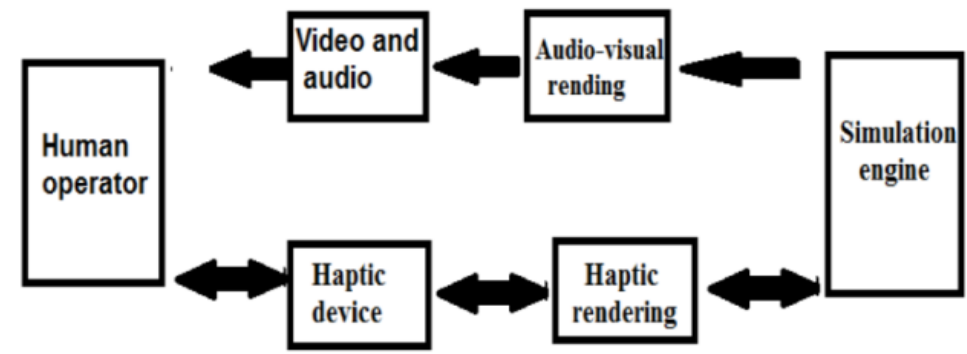

Fig 2 haptic feedback

Virtual reality (VR) applications strive to simulate real or imaginary scenes with which users can interact and perceive the effects of their actions in real time.Figure-3 below shows the structure of a VR application incorporating visual, auditory, and haptic feedback [9].

Here in this case already we have used a servo motor for each finger but in this case we will face some problems i.e. we can't grab the object perfectly. Now we can use a joint technique in which the finger should have several joints and the movement of joints can be controlled by using inverse kinematics. By using inverse kinematics we can analyze the finger joint movement. Our finger has generally there are three joints and if we can provide three joints to the finger of robotic arm then it will be easy to grab the project. Here we can use micro motor for each joint.

Before this in the system there is no camera for detecting the object. But we can add a camera and can detect the object by using RGB method and we can specify some object and we the robot can detect the object by comparing saved object.

\subsection{A five fingered robotic arm:}

\section{DESIGN OF FIVE FINGERED ROBOTIC ARM}

The motion to the robotic arm is given by servo motors. Servo motors control the motion of each joint of the robotic arm. The DOF of the robotic arm depends up on the placement of servo motors. The maximum load that this robotic arm can survive depends on the rating of servo motors. Likewise the fingers of robotic arm made of servo motors. The DOF of the robotic arm and fingers are limited due to use of servo motors. But the movement of the robotic arm is very much similar to that of human hand. This movement is controlled by a sensor based glove which is mounted on the controller's hand. Working of virtual robotic system in block diagram:

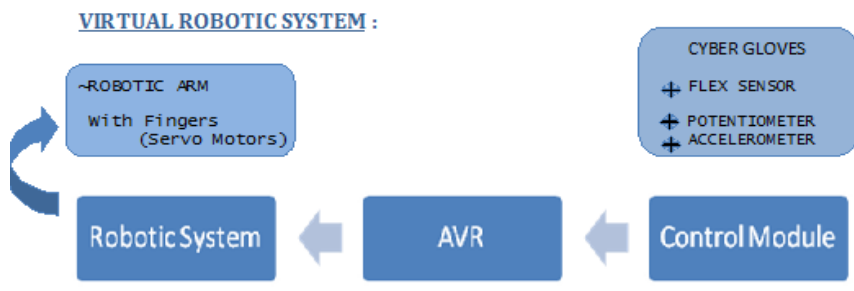

Fig.3 virtual robotic system

For each joint of finger we can use separate motors. The flex sensor connected at each finger, when bends sends 3 analog values to AVR board as there are three joints in a finger and according to the values the motors rotate.

\subsection{Servo Motors}

A closed-loop motion control system, as shown in block diagram has one or more feedback loops that continuously compare the system's response with input commands or settings to correct errors in motor and/or load speed, load position, or motor torque. Feedback sensors provide the electronic signals for correcting deviations from the desired input commands. 


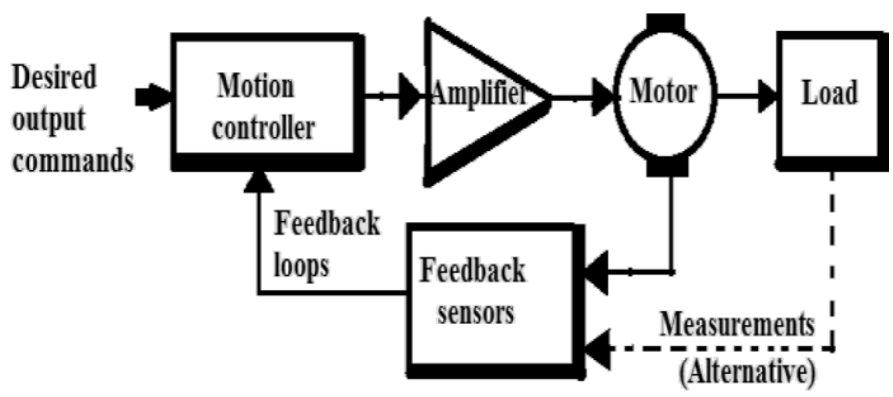

Fig 4 closed loop system

\subsection{Glove based controlling unit:}

All sensors are variable resistive type. These sensors are mounted on a glove so that the whole sensing unit will give different analog outputs as per the controller's hand movement. These analog outputs control the movement of servo motors. The sensors are described below,

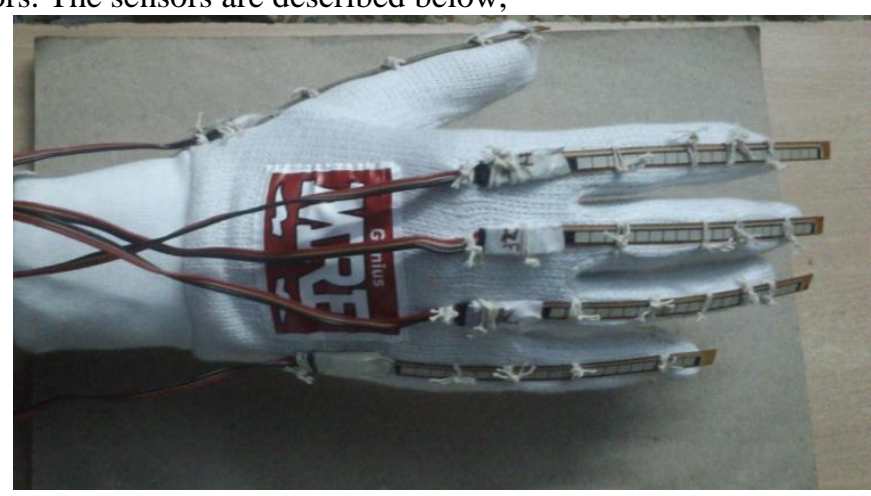

Fig 5 design of five fingered hand

\subsection{Accelerometer Sensor / 3-axis gyro sensor:}

Accelerometer sensor can measure static (earth gravity) or dynamic acceleration in all three axes. Application of the sensor is in various fields and many applications can be developed using this sensor. Sensor measures level of acceleration where it is mounted this enable us to measure acceleration/deceleration of objects. Acceleration is a vector force which has direction and measured in meters per second.

\subsection{Flex sensor / Bend Sensor:}

The Flex Sensor patented technology is based on resistive carbon Elements. As a variable printed resistor, the Flex Sensor achieves great form-factor on a thin flexible substrate. When the substrate is bent, the sensor produces a resistance output correlated to the bend radius the smaller the radius, the higher the resistance value.

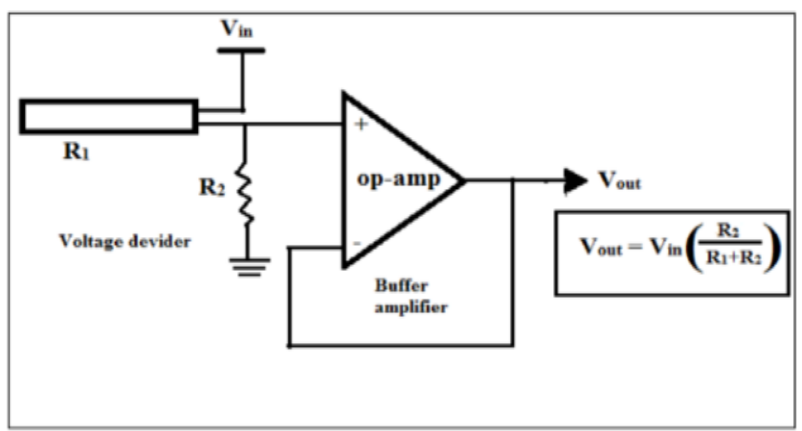

Figs 6 flex sensor circuit 


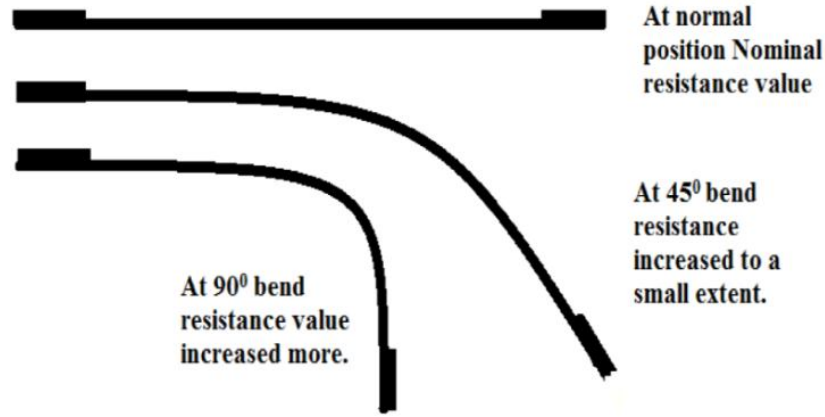

Fig 7 flex sensor offer various resistance

\subsection{Potentiometer:}

Potentiometer can give different analog value, by taking those values we can operate different functions of robotic arm using AVR board, where the output of potentiometer is given to AVR board.

\subsection{Controlling Servo Motors:}

\section{TECHNOLOGY}

For controlling servo motors pulse of different duty cycles need to be provided. These pulses can be generated as PWM signals. Using AVR boards we can generate PWM signals by manipulating timer based interrupt.

Working: Ton+Toff $=20 \mathrm{~ms}$

Ton should vary from 1 to $2 \mathrm{~ms}$

Use two interrupt compare match interrupt A. It should trigger on each $20 \mathrm{~ms}$ and compare match interrupt B it should trigger before 1 or 2 millisecond of triggering compare match interrupt A. In compare match interrupt B reset the port pin and again in compare match interrupts A set the port pin. This set and reset of the port pin should be done manually in the ISR.

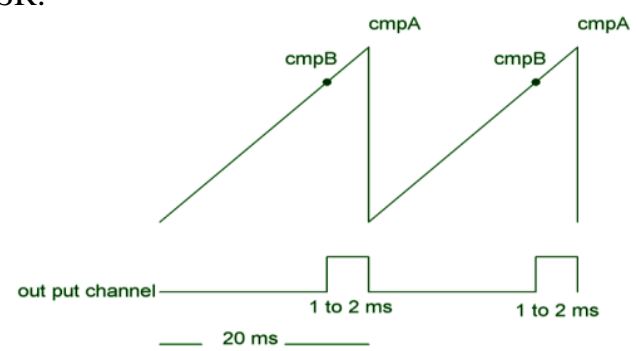

Fig 8 controlling of single servo motor

\subsection{Kinematics:}

Here for analysis of joints in a finger we are using forward and inverse kinematics. Inverse kinematics refers to the use of the kinematics equations of a robot to determine the joint parameters that provide a desired position of the end-effectors. Specification of the movement of a robot so that its end-effectors achieve a desired task is known as motion planning. Inverse kinematics transforms the motion plan into joint actuator trajectories for the robot. The movement of a kinematic chain whether it is a robot or an animated character is modeled by the kinematics equations of the chain. These equations define the configuration of the chain in terms of its joint parameters. Forward kinematics uses the joint parameters to compute the configuration of the chain, and inverse kinematics reverses this calculation to determine the joint parameters that achieves a desired configuration. 

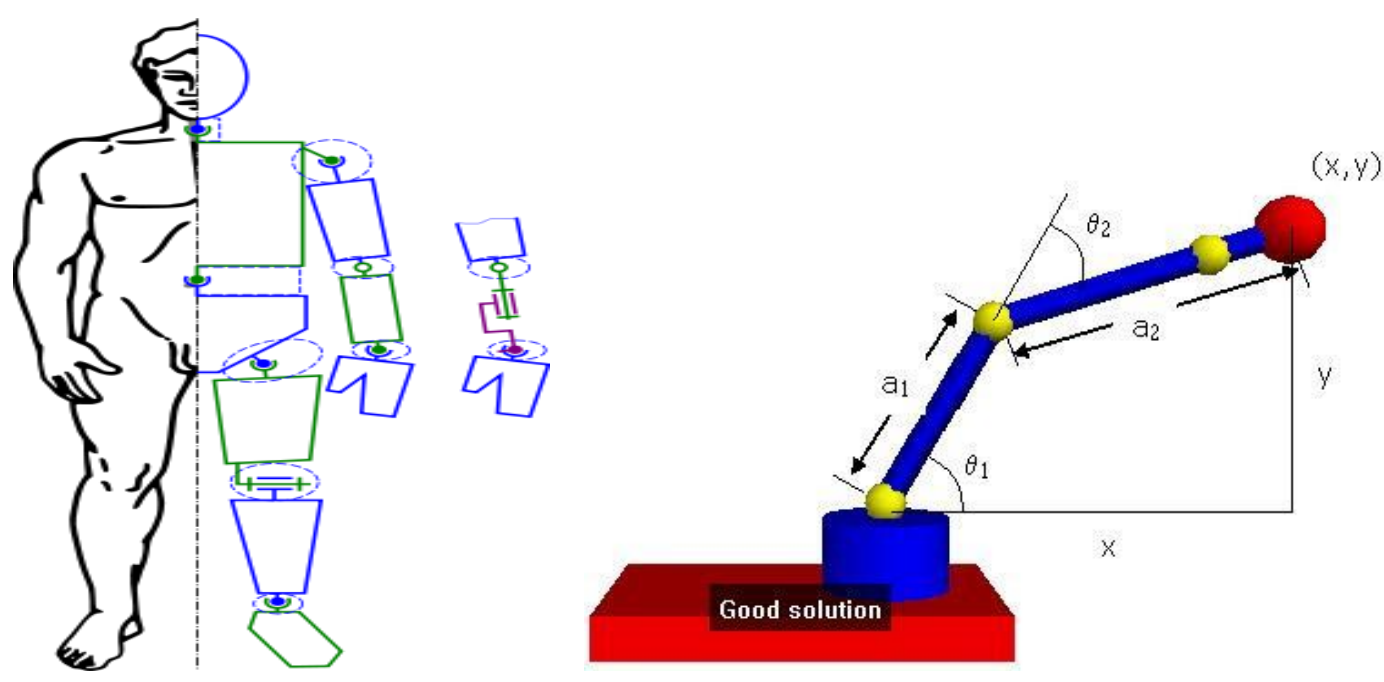

Fig 9 A model of the human skeleton as a kinematic chain allows positioning using inverse kinematics and a example of forward kinematics.

For example, inverse kinematics formulas allow calculation of the joint parameters that position a robot arm to pick up a part. Similar formulas determine the positions of the skeleton of an animated character that is to move in a particular way.

Inverse kinematics is an example of the kinematic analysis of a constrained system of rigid bodies, or kinematic chain. The kinematics equations of a robot can be used to define the loop equations of a complex articulated system. These loop equations are non-linear constraints on the configuration parameters of the system. The independent parameters in these equations are known as the degrees of freedom of the system.

Forward kinematics refers to the use of the kinematic equations of a robot to compute the position of the endeffector from specified values for the joint parameters. The kinematics equations of the robot are used in robotics, computer games, and animation.

\subsection{Sensing and ADC Manipulation:}

Each tactile sensors give analog values which can be given as input to the controller as ADC value. These ADC values control the PWM pulse generation which will be given as input to the servo motors.

\subsection{Microcontroller:}

For generating different pulses for controlling servo motor movement a processor / microcontroller is used. Hex file of C-code is downloaded to the controller and it performs the task accordingly. The code is written in such a way that it takes the analog output of the sensors as input and according to the ADC value of that input it generates pulses of different duty cycles. So basically it performs the as that of human brain, which give the motion to the robotic arm according to the movement by the human hand.

\section{GRAPH AND SIMULATION}

All the graphs that have been given depend up on the coding that has been downloaded to the microcontroller. The linearity between input and output depends on the programming. The range of input and output also depends on the programming.

\subsection{Sensor output for servo motor (potentiometer):}

Here the analysis is done between output of the potentiometer and output of the servo motor. 

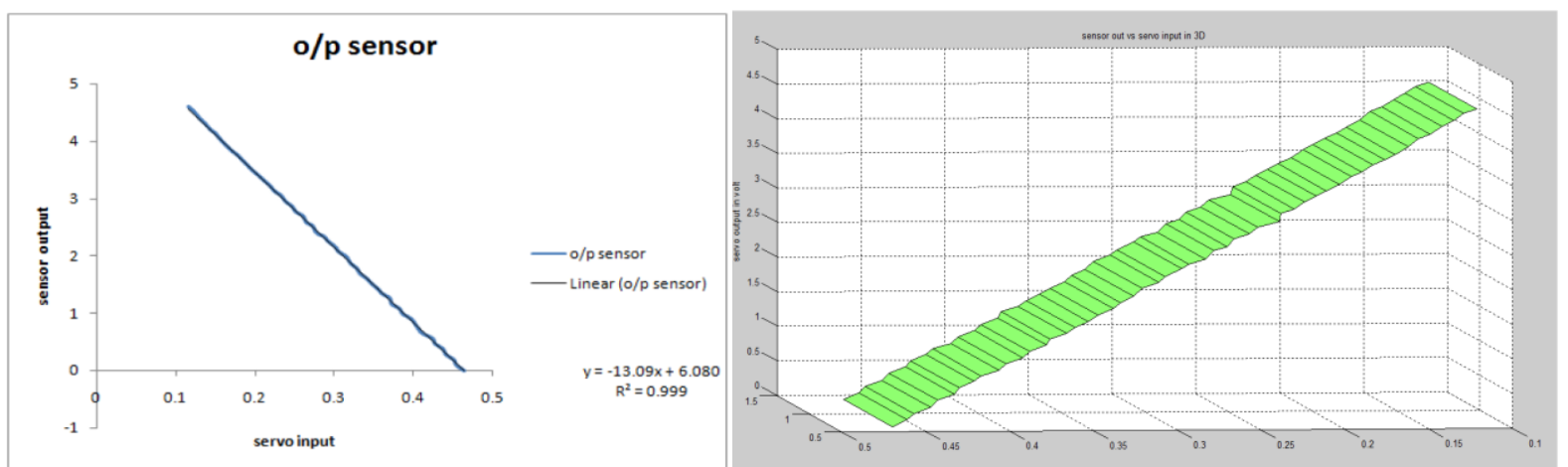

Fig 10 output voltage of potentiometer with respect to the input voltage of servo motor.

From the above graph R2 is found to be 0.99 and slope is found to be -13.09 and respective equation is in the figure.

\subsection{Sensor output vs. servo motor (flex sensor):}

Here the analysis is done between output of the flex sensor and output of the servo motor.

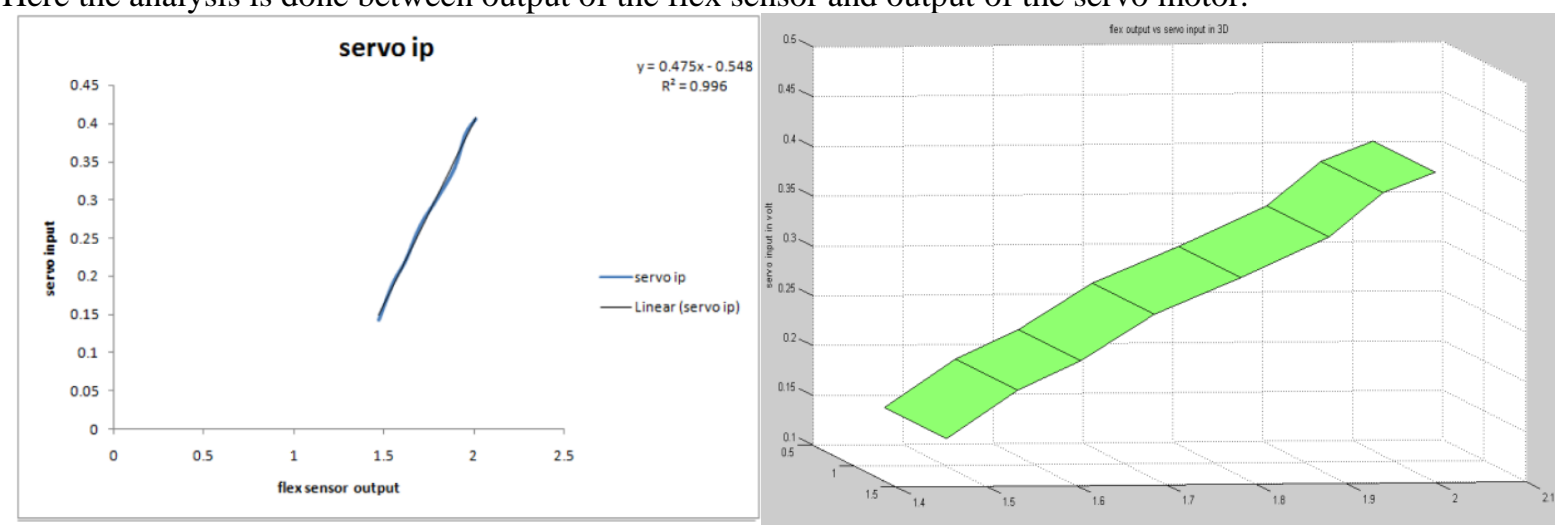

Fig 11 output voltages of flex sensor respect to the input voltage of servo motor.

From this graph also R2 is found to be 0.996 and the slope is 0.475 and the respective equation is in the figure.

\subsection{Output of potentiometer to servo motor in terms of angle:}

Here the analysis is done between output of the potentiometer and the angle moved by the servo motor.

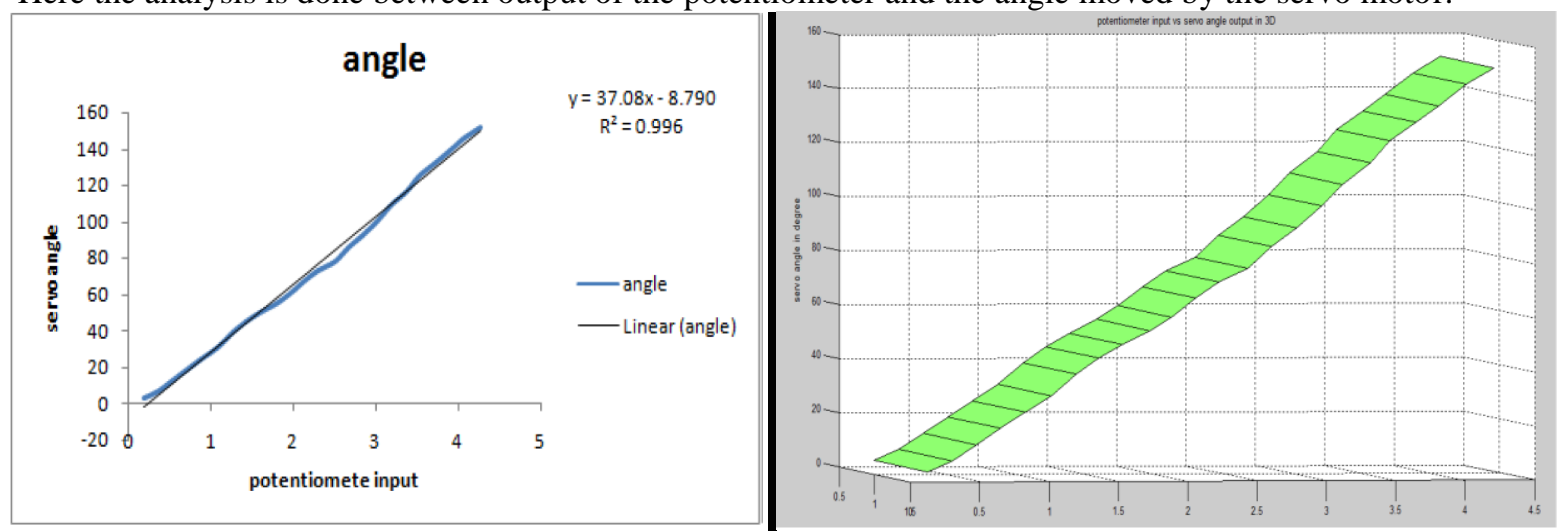

Fig 12 servomotor angle respect to potentiometer input voltage

From this graph R2 is found to be 0.996 and the slope of the linear graph is found to be 37.08 and the respective equation is in the graph

4.4 Output of forward kinematics as position orientation matrix:

Here the analysis is done of the output of the 4 joint-angles of robot finger of an arm. 

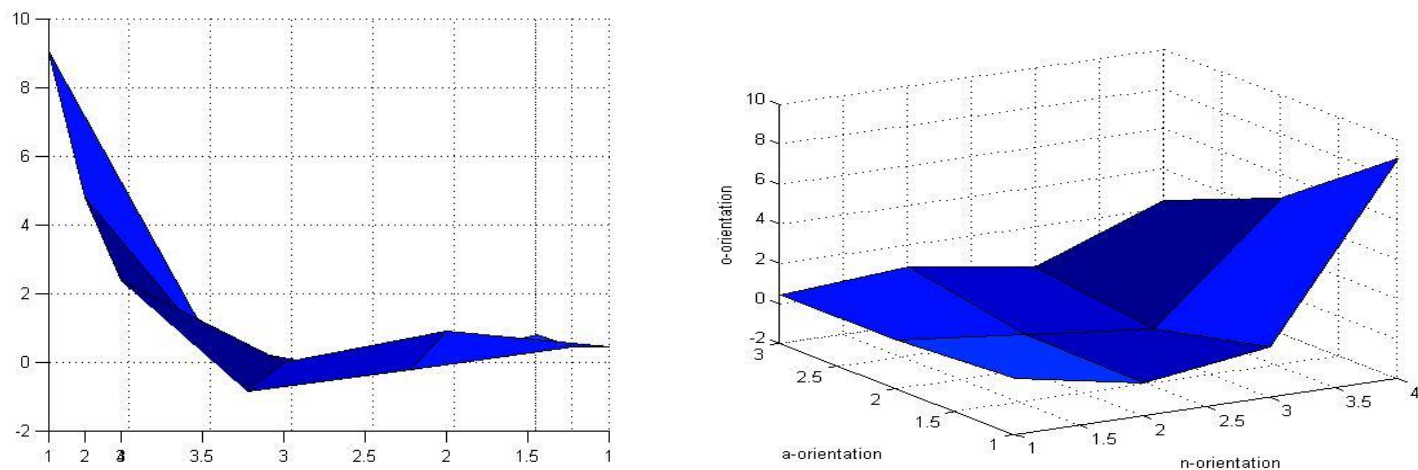

Fig 13 an outputs of 4 D.O.F joint angles of finger of a robotic arm

\section{CONCLUSION}

In this paper we developed a five fingered haptic arm with flex sensor and servomotor. This reduces the number of wires in the design method, increases compactness, and improves the transportability of the total interface. We carried out some experiments on different movement of haptic arm and measured sensor output and servo motor angle. The analog outputs of sensor are manipulated in such a way that it will give pulse of different duty cycles to the servo motor which will control the motion of servo motor. The sensor gives analog output, if there is a change in their resistance value. This analog output is manipulated in such a way that they give proportional pulses of different duty cycles to the servo motors which give movement to the robotic arm. The analog outputs of sensor are manipulated in such a way that it will give pulse of different duty cycles to the servo motor which will control the motion of servo motor and we can grab an object perfectly as we are using joints in the robotic finger.

\section{REFERENCES}

[1]. Takahiro Endo, Haruhisa Kawasaki, Tetsuya Mouri, ,Yasuhiko Ishigure,Hisayuki Shimomura,Masato Matsumura,andKazumi Koketsu, || Five-Fingered Haptic Interface Robot:HIRO III || IEEE transactions on haptics, vol. 4, no. 1, january-march 2011.

[2]. Hyun Soo Woo and Doo Yong Lee,\| Exploitation of the Impedance and Characteristics of the Human Arm in the Design of Haptic Interfaces\| IEEE transactions on industrial electronics, vol. 58, no. 8, august 2011.

[3]. J. E. Colgate and G. Schenkel, -Passivity of a class of sampled-data systems: Application to haptic interfaces, || J. Robot. Syst., vol. 14, no. 1,pp. 37-47, 1997.

[4]. G. C. Burdea, Force and Touch Feedback for Virtual Reality. New York: Wiley, 1996.

[5]. D.K.Boman,-Internationalsurvey:Virtual-environment research,\| Computer, vol. 28, no. 6, pp. 5765, Jun. 1995.

[6]. S.D. Lay and A.M.Day, - Recent developments and applications of haptic devices, $\|$ Comp. Graph. Forum, vol. 22, no. 2, pp. 117-132, 2003.

[7]. Ninja P. Oess, ,Wanek, J. and van Hedel, H.J.A —Enhancement of bend sensor properties as applied in a glove for use in neurorehabilitation settings\|, Engineering in Medicine and Biology Society (EMBC), 2010 Annual International Conference of the IEEE, Aug. 31 2010-Sept. 4 2010,

[8]. J. E. Colgate and J. M. Brown, -Factors affecting the Z-width of a haptic display, $\|$ in Proc. IEEE Int. Conf. Robot. Autom., San Diego, CA, 1994, pp. 3205-3210.

[9]. Li Jiang, Member, IEEE, Mark R. Cutkosky, Juhani Ruutiainen, and Roope Raisamo, \|I Using Haptic Feedback to Improve Grasp Force Control in Multiple Sclerosis Patients\| IEEE transactions on robotics, vol. 25, no. 3 , june 2009 .

[10]. Laura Dipietro, Angelo M. Sabatini, and Paolo Dario, -A Survey of Glove-Based Systems and Their Applications\| IEEE transactions on systems, man, and cybernetics-part c: applications and reviews, vol. 38, no. 4, july 2008.

[11]. Junseok Chae, Haluk Kulah, and Khalil Najafi, \| A Monolithic Three-Axis Micro-g Micromachined Silicon Capacitive Accelerometer\|, journal of microelectromechanicalsystems, vol. 14, no. 2, april 2005.

[12]. William R. Provancher, Mark R. Cutkosky,dexterous manipulation lab, Katherine J. Kuchenbecker, Gunter Niemeyer, Telerobotics lab, Stanford University, || Contact Location display for Haptic Perception Of Curvature and 